\title{
A Peculiar Case of Abdominal Pain in an Eleven Years Old Child
}

\author{
Sahbaa Fehr
}

Lecturer of pediatrics, Zagazig University, Egypt

Corresponding author: Sahbaa Fehr, Lecturer of pediatrics, Zagazig University, Egypt, Tel: +201208112002; Fax: 1208112002; E-mail: sahbaafehr@yahoo.com

Received: 26 December 2016; Accepted: 02 March 2017; Published: 06 March 2017

Citation: Fehr S. A Peculiar Case of Abdominal Pain in an Eleven Years Old Child. Ann Clin Lab Res. 2017, 5: 1.

\section{Abstract}

An 11 years old boy presented to us with three weeks history of severe colicky abdominal pain and dark colored urine. It would not be relieved by anti-colic medication. This attack of abdominal pain was associated with dark colored urine with hypertension and anemia. There was a past history of recurrent abdominal pain that started at the age of 3 years old. This Pain occurred in attacks that would last for days, these attacks were afebrile not associated with diarrhea, vomiting, rash, jaundice or dark urine.

Initially these attacks would be relieved by Parasympatholytic drugs, the patient then would have free months then the pain would recur. Investigations showed $\mathrm{Hb} 9 \mathrm{mg} / \mathrm{dl}$, ESR 70, CRP 55, negative ANA, negative anti DNAse, urine RBCS over 100, normal HB electrophoresis, the patient was MEFV gene mutation double heterozygous. It was atypical case of familial medientranian fever.

Keywords: Abdominal pain; No fever; Dark urine; MEFV mutation

Abbreviations: HB: Hemoglobin; ESR: Erythrocyte Sedimentation Rate; CRP: C Reactive Protein; ANA: Antinucleoside Antibody; FMF: Familial Medientranian Fever

\section{Case Presentation}

An 11 years old boy presented with three week's history of severe colicky abdominal pain and dark colored urine. The abdominal pain was mainly epigastric and periumblical without propagation. The pain has no diurnal variation, it was not associated with vomiting or diarrhea, it was not related to meals or fasting. It would not be relieved by anti-colic medication. There was no abdominal enlargement, no jaundice, no dysphagia and no loss sphincteric control.

This attack of abdominal pain was associated with dark colored urine with hypertension and anemia. There was no edema, no polyuria or oliguria, no skin rash. There was no history suggesting other system affection.
A diagnosis of acute glomerulonephritis was suggested. However, the condition cannot be taken as simple as acute glomerulonephritis. There was a past history of recurrent abdominal pain that started at the age of 3 years old. This Pain occurred in attacks that would last for days; these attacks were afebrile not associated with diarrhea, vomiting, rash, jaundice or dark urine. Initially these attacks would be relieved by Parasympatholytic drugs, the patient then would have free months then the pain would recur.

Investigations showed $\mathrm{Hb} 9 \mathrm{mg} / \mathrm{dl}$, ESR 70, CRP 55, negative ANA, negative anti DNAse, urine RBCS over 100, normal HB electrophoresis.

\section{Discussion}

By the time of presentation that patient was stamped for many years by different diagnosis for which he received treatment but was not successful, for example he was diagnosed for those attacks as rheumatic fever and received long acting penicillin, parasitic infestations were also considered.

For us we considered many differentials first a vasculitis disease because of Nephritis Abdominal pain, Anemia, Elevated acute phase reactants, yet there were No other criteria: arthritis, malar rash, discoid rash, carditis, photophobia, oral ulcer, neurological signs. we ordered ANA, anti-DNase which were negative.

We considered sickle cell anemia because of Anemia, Spleenomegaly Recurrent abdominal pain, nephritis yet against it Anemia was microcytic, No history of blood transfusion. We ordered diagnostic hemoglobin electrophoresis which was normal.

Finally, atypical FMF was considered as patient had recurrent abdominal pain, what was atypical is Red urine, absent fever, No leucocytosis, Discrepancy between severity of complaint and sparsity of clinical findings.

However genetic testing showed the patient was double heterozygous for FMF gene mutation "MEFV.M694V".

Familial Mediterranean fever is an autosomal recessive genetic disorder characterized by recurrent febrile polyserositis, especially prevalent in individuals of Mediterranean descent. Familial Mediterranean fever can have nonspecific manifestations that mimic many common acquired 
disorders such as infections, acute appendicitis, cholecystitis, and arthritis, which can delay diagnosis for many years and subject patients to extensive evaluations and even unnecessary surgery [1].

The gene locus responsible for FMF was identified in 1992 and localized to the short arm of chromosome 16. In 1997, a specific FMF gene locus, MEFV, was discovered to encode for a protein, pyrin that mediates inflammation. To date, more than forty missense mutations are known to exist. The diversity of mutations identified has provided insight into the variability of clinical presentation and disease progression [2].

The function of pyrin has not been completely elucidated, but it appears to be a suppressor of the activation of caspase 1 , the enzyme that stimulates production of interleukin $1 \beta$, a cytokine central to the process of inflammation. In other words, an ineffective pyrin doesn't inhibit inflammation normally, resulting in inflammatory episodes of membranes at differing sites. There are seven types of attacks. Ninety percent of all patients have their first attacks before they are 18 years old. All develop over 2-4 hours and last anywhere from 6 hours to 4 days. Most attacks involve fever. Abdominal attacks, featuring abdominal pain, affect the whole abdomen with all signs of peritonitis (inflammation of abdominal lining), and acute abdominal pain like appendicitis. They occur in $95 \%$ of all patients and may lead to unnecessary laparotomy.

\section{Complications}

AA-amyloidosis with kidney failure is a complication and may develop without overt crises. AA amyloid protein is produced in very large quantities during attacks, and at a low rate between them, and accumulates mainly in the kidney, as well as the heart, spleen, gastrointestinal tract and thyroid [3].

Renal manifestations in familial Medientranian fever: IN FMF due to impaired control of inflammatory process this predisposes to vasculitis like PAN and HSP and renal. On microscopic examination of the kidney specimens, some patients were found to have amyloidosis, others focal segmental glomerulosclerosis and few patients with membranoproliferative glomerulonephritis [4].

The diagnosis is clinically made on the basis of the history of typical attacks, especially in patients from the ethnic groups in which FMF is more highly prevalent. An acute phase response is present during attacks, with high C-reactive protein levels, an elevated white blood cell count and other markers of inflammation. A genetic test is also available to detect mutations in the MEFV gene. Sequencing of exons 2,3,5 and 10 of this gene detects an estimated $97 \%$ of all known mutations [6].

\section{Treatment}

Attacks are self-limiting, and require analgesia and NSAIDs (such as Diclofenac).

It is compulsory to treat symptomatic FMF patients for preventing the acute attacks and the development and progression of secondary (AA) amyloidosis. Colchicine, a drug otherwise mainly used in gout, decreases attack frequency in FMF patients. The exact way in which Colchicine suppresses attacks is unclear. While this agent is not without side effects (such as abdominal pain and muscle pains), it may markedly improve quality of life in patients. The dosage is typically 1 $\mathrm{mg} /$ day to $2 \mathrm{mg} /$ day. Development of amyloidosis is delayed with Colchicine treatment [7].

The biological target of colchicine is tubulin, a globular protein involved in the composition of microtubules. Colchicine blocks tubulin polymerization interfering white blood cells migration and degranulation. Also, colchicine interferes with activation of NALP3 (Nacht domain-, leucinerich repeat-, and PYD-containing protein 3, also called cryopyrin) inflammasome. Colchicine has an oral bioavailability of $50 \%$ and is absorbed in the jejunum and ileum into the enterohepatic circulation. A second peak of serum concentration is generally observed 5-7 hours later. After a single i.v. injection, colchicine plasma half-life is $20 \mathrm{~min}$, while after oral administration is $60 \mathrm{~min}$. Colchicine is excreted mainly by hepatic metabolism via oxidative demethylation by the cytochrome P450 system (isoform CYP 3A4). Metabolites of colchicine are 2- and 3-demethylcolchicine [8]

Other therapeutic agents such as IL-1 $\beta$ inhibitors (anakinra, rilonacept, canakinumab) have been proposed in colchicineresistant or colchicine-intolerant patients. Anakinra inhibits the IL-1 proinflammatory pathway binding IL- $1 \alpha$ and IL-1 $\beta$ to the IL-1 receptor type I effects include local pain and erythema due to subcutaneous injection, and the major complications are acute interstitial pneumonia], neutropenia grade II acute rejection according to Banff classification and also hypertension requiring therapy [9].

\section{References}

1. Zadeh N, Getzugand T, Grody W (2011) Diagnosis and management of familial Mediterranean fever: Integrating medical genetics in a dedicated interdisciplinary clinic. Genet Med 13: 263-69.

2. Fisher PW, Ho LT, Goldschmidt R, SemerdjianRJ, Rutecki GW (2003) Familial Mediterranean fever, inflammation and nephrotic syndrome: Fibrillary glomerulopathy and the M680I missense mutation. BMC Nephrology 4: 6.

3. van der Hilst JC, Simon A, Drenth JP (2005) Hereditary periodic fever and reactive amyloidosis. ClinExp Med 5: 87-98.

4. Bakkaloglu A (2003) Familial Mediterranean fever. PediatrNephrol 18: 853-859.

5. Portincasa P, Scaccianoce G, Palasciano G (2013) Familial mediterranean fever: A fascinating model of inherited autoinflammatory disorder. Eur J Clin Invest 43: 1314-1327.

6. Wang DQ, Bonfrate L, de Bari O, Wang TY, Portincasa P (2014) Familial Mediterranean fever: From pathogenesis to treatment. J Genet Syndr Gene Ther 5: 248.

7. Lidar M, Scherrmann JM, Shinar Y, Chetrit A, Niel E, et al. (2004) Colchicine non-responsiveness in familial Mediterranean fever: clinical, genetic, pharmacokinetic, and socioeconomic characterization. Semin Arthritis Rheum 33: 273-282. 
8. Cerquaglia $C$, Diaco $M$, Nucera $G$, La Regina $M$, Montalto $M$, et al. (2005) Pharmacological and clinical basis of treatment of familial Mediterranean fever (FMF) with colchicine or analogues: An update. Curr Drug Targets Inflamm Allergy 4: 117-124.
9. Moser C, Pohl G, Haslinger I, Knapp S, Rowczenio D, et al. (2009) Successful treatment of familial Mediterranean fever with Anakinra and outcome after renal transplantation. Nephrol Dial Transplant 24: 676-678. 